\title{
Feasibility of the EQ-5D in the elderly population: a systematic review of the literature
}

\author{
Ole Marten $^{1}$ (D) Laura Brand ${ }^{1} \cdot$ Wolfgang Greiner $^{1}$ (D)
}

Accepted: 26 September 2021 / Published online: 6 October 2021

(c) The Author(s) 2021

\begin{abstract}
Purpose The EQ-5D-3L and 5L are widely used generic preference-based instruments, which are psychometrically sound with the general population, but little is known about the instruments' feasibility in the elderly. Therefore, this systematic review summarises the available literature with regard to the feasibility properties of the instruments in the elderly population. Methods We conducted a systematic search in PubMed, PsycInfo and EuroQol databases using pre-specified vocabulary and inclusion/exclusion criteria to identify publications until November 2020. Study characteristics and outcomes referring to the feasibility of the EQ-5D-3L and 5L in the elderly were extracted, if all study participants were at least 65+ years.

Results We identified 17 studies reporting feasibility outcomes based on four criteria: missing values, completion rates, completion time and broad qualitative statements referring to the completion. Missing values per dimension ranged from 0 to $10.7 \%$, although being mostly below $7 \%$. The completion rate was around $90 \%$ or better, whereas the EQ VAS rating was missing from 2.3 to $25.3 \%$ of the respondents. Only two of the included studies examined the EQ-5D-5L; 15 studies reported on the EQ-5D-3L.

Conclusion Comparing our findings against the general population from published literature, we find that feasibility outcomes in older age groups are just below that of younger populations. Furthermore, older respondents have a higher propensity of requiring assistance or even an interviewer-based approach. Nonetheless, the reviewed literature indicates that the EQ-5D-3L still has good feasibility properties and, hence, is highly applicable in older respondents. However, further research is needed to explore feasibility properties of the EQ-5D-5L in this population.
\end{abstract}

Keywords EQ-5D $\cdot$ EQ-5D-5L $\cdot$ Health-related quality of life $\cdot$ Systematic review $\cdot$ Feasibility $\cdot$ Elderly

\section{Background}

In the past decades, the demographic trend of an ageing population has become one of society's central challenges. Especially the proportion of the elderly population, i.e. people being 65 years and above [1], is growing faster than any other age group [2]. At the same time, the increase in life expectancy induces a higher individual risk of contracting one or multiple diseases over the course of life, eventually resulting in a growing number of multi-morbid patients with chronic diseases [3]. Chronic illness and multi-morbidity are known to be associated with disability, declined functional

Ole Marten

ole.marten@uni-bielefeld.de

1 Department of Health Economics and Health Care Management, School of Public Health, Bielefeld University, Universitaetsstrasse 25, Bielefeld, Germany status and diminished quality of life (QoL) [4], which is further linked to higher health care utilisation and increased costs of health care [5]. Given the natural limit of health care resources, it is necessary to evaluate the cost-effectiveness of health care interventions from a societal perspective to sustain the health service provision [6].

Health-related quality of life (HRQoL) is a central outcome for the benefit assessment of health and social care interventions often measured as patient reports to gather the patients' subjective assessment of their health condition. The EQ-5D is a generic preference-based measure of HRQoL to operationalise quality-adjusted life years (QALYs) in economic evaluation [7]. The EQ-5D consists of two sections. The first is a descriptive system covering five dimensions: mobility (MO), self-care (SC), usual activities (UA), pain or discomfort (PD) and anxiety or depression (AD). In the EQ5D-3L each dimension can be described by three severity levels (1-no problems; 2 -moderate problems; 3 -unable 
to), thus allowing to distinguish 243 unique health states $\left(3^{\wedge} 5\right)$. The second part is a visual analogue scale (EQ VAS) - a vertical thermometer-ranging from 0 ('worst imaginable health') to 100 ('best imaginable health') gauging the respondent's subjectively rated health which might cover aspects different from those in the descriptive system $[8,9]$. A later variant, the EQ-5D-5L, covers the same five dimensions, but allowing the respondent to choose from five response levels, thus describing 3125 unique health states [10]. For both versions of the EQ-5D the descriptive system can be scored using a tariff, which provides the preference weights for each health state allowing to calculate an index value on the 0-1 QALY scale, whereas the EQ VAS rating is commonly analysed independently from the responses to the descriptive system [11].

The EQ-5D (hereafter, used to refer to both the EQ5D-3L and EQ-5D-5L) has been shown to be applicable in various health conditions and populations and is the most widely used instrument for use in economic evaluation [12-14]. Literature suggest that the EQ-5D is also frequently used in the economic evaluation of interventions for the elderly population [15-19]. However, as of yet there is no established gold standard as to how HRQoL in the elderly should ultimately be measured. Especially with regard to the elderly population further requirements to the appropriate assessment of HRQoL are made, since people of higher age may be different to their younger counterparts in the general population in terms of their physical or mental abilities, education or understanding of health $[17,20]$. Common criteria to evaluate an instrument's performance are its reliability, validity as well as its feasibility [21]. Generally, the EQ-5D's measurement properties are well examined [22, 23] with several studies specifically confirming the instrument's reliability and validity in the older population, however, leaving the feasibility property widely untouched and warranting further research [18, 24, 25]. In this sense, feasibility is concerned with the difficulty or ease of applying the measure in a population, which translates into how well the measure is regularly completed [21, 26, 27]. Beyond these descriptions there is no gold standard to the definition or operationalisation of feasibility with regard to HRQoL measures. A prior search across all age groups indicates that the feasibility of the EQ-5D is typically associated with the proportion of missing values [13, 28-31], time required for completion and the appropriateness of the administration mode [26, 32].

Age-related decline may be an obstacle for elderly respondents when self-reporting their HRQoL in a survey $[33,34]$. Therefore, offering interviewer support or administration can help to reduce the burden to respondents and thereby have a positive impact on item response [35, 36]. There is recent qualitative evidence from older $(60+$ years $)$ hip fracture patients suggesting that interviewer support was needed and had a positive effect on the instruments' completion [37]. Even though the EQ-5D is a short measure there appears to be some demand for interviewer support, however, it is unclear how commonly this is applied when collecting EQ-5D data in the elderly.

Despite the steadily growing population of those aged 65 years and above, to date little is known about the feasibility of the EQ-5D in the elderly. Therefore, the aims of this literature review were as follows: (i) to assess the feasibility properties of the EQ-5D in the elderly and (ii) to examine the role of interviewer support in collecting EQ-5D data in samples of the elderly population.

\section{Methods}

\section{Literature search}

We conducted a systematic literature search up until June 2019 with the aim to identify all studies in either English or German assessing the feasibility of the EQ-5D-3L and 5L in the elderly population. We performed electronic searches in the PubMed and PsycINFO (EBSCO) databases as well as the EuroQol Research Foundation Website [38] to identify publications of interest. The search involved MeSH terms and synonymous free-text terms around the following keywords "EQ-5D", "elderly" and "feasibility". Additionally, a manual search was carried out based on the reference lists of included studies. Detailed information on the search strategy can be found in the appendix. Articles were included if they met the following inclusion criteria: (1) application of the EQ-5D-3L or $5 \mathrm{~L}$ as a primary or secondary outcome measure; (2) the minimum age of the sample is specifically reported and it does not include participants younger than 65 years of age and (3) information on the feasibility of the EQ-5D-3L or 5L was reported at least as secondary analysis. Articles were excluded if they were not in English or German, not available in full text or of a wrong publication type, i.e. abstracts, proceedings, review articles and study protocols. No restrictions were imposed on the cognitive status of study participants. The literature search was updated in November 2020 applying the same criteria as outlined above.

\section{Screening and data extraction}

Two reviewers (OM and $\mathrm{LB}$ ) independently screened the title and abstract of all identified studies after electronic and manual removal of duplicates. At the end of each screening stage, discrepancies were discussed and, if necessary, a third reviewer (WG) was consulted to resolve any variance. Subsequently, full texts for appropriate articles were retrieved and assessed for eligibility based on pre-defined inclusion and exclusion criteria. A standardised data extraction form 
was used to guide a structured review process. The data extraction process was conducted by one reviewer (LB) and verified by the second reviewer (OM) to check for missing extractions. The following information was extracted for each study: author, year of publication, country, study type, EQ-5D version, mode of administration, number and age of participants. We further extracted characteristics, which are specific to older adults such as information on the functional status and the living arrangement.

During the search and extraction process of information related to the feasibility of the EQ-5D, we concentrated on information regarding the proportion and distribution of missing responses of both the descriptive system and EQ VAS to identify potentially problematic items. Derived from that, we examined the completion rate describing the proportion of computable index values, which is only viable if the EQ-5D health state information is complete on all five dimensions (excluding the EQ VAS) [11, 39]. We further screened for information on the time needed to complete all components of the EQ-5D, the administration mode as well as its appropriateness. Nonetheless, related aspects such as the ability to complete the measurement or problems during data collection process were also extracted.

\section{Results}

\section{Study selection}

Our initial search including articles until June 2019 retrieved 2063 articles from the PubMed, PsycInfo and EuroQol website databases; 12 additional references were identified during the manual search of reference lists. After removal of duplicates, 1766 references were screened based on their titles and abstracts resulting in the exclusion of 1613 references. The remaining 153 studies were screened for eligibility in full text. Of those, 139 studies did not meet the inclusion criteria and, hence, were excluded. Main reasons for exclusion were the unavailability of feasibility information, insufficient age (sample not exclusively 65 years and over; only mean age reported) and publication type (no full text available; review article). The remaining 14 articles included in the review focus on aspects of the EQ-5D's feasibility in the elderly population. The further conducted review update, including articles published between June 2019 and November 2020, resulted in three additional hits. Thus, the review eventually included 17 articles (Fig. 1).

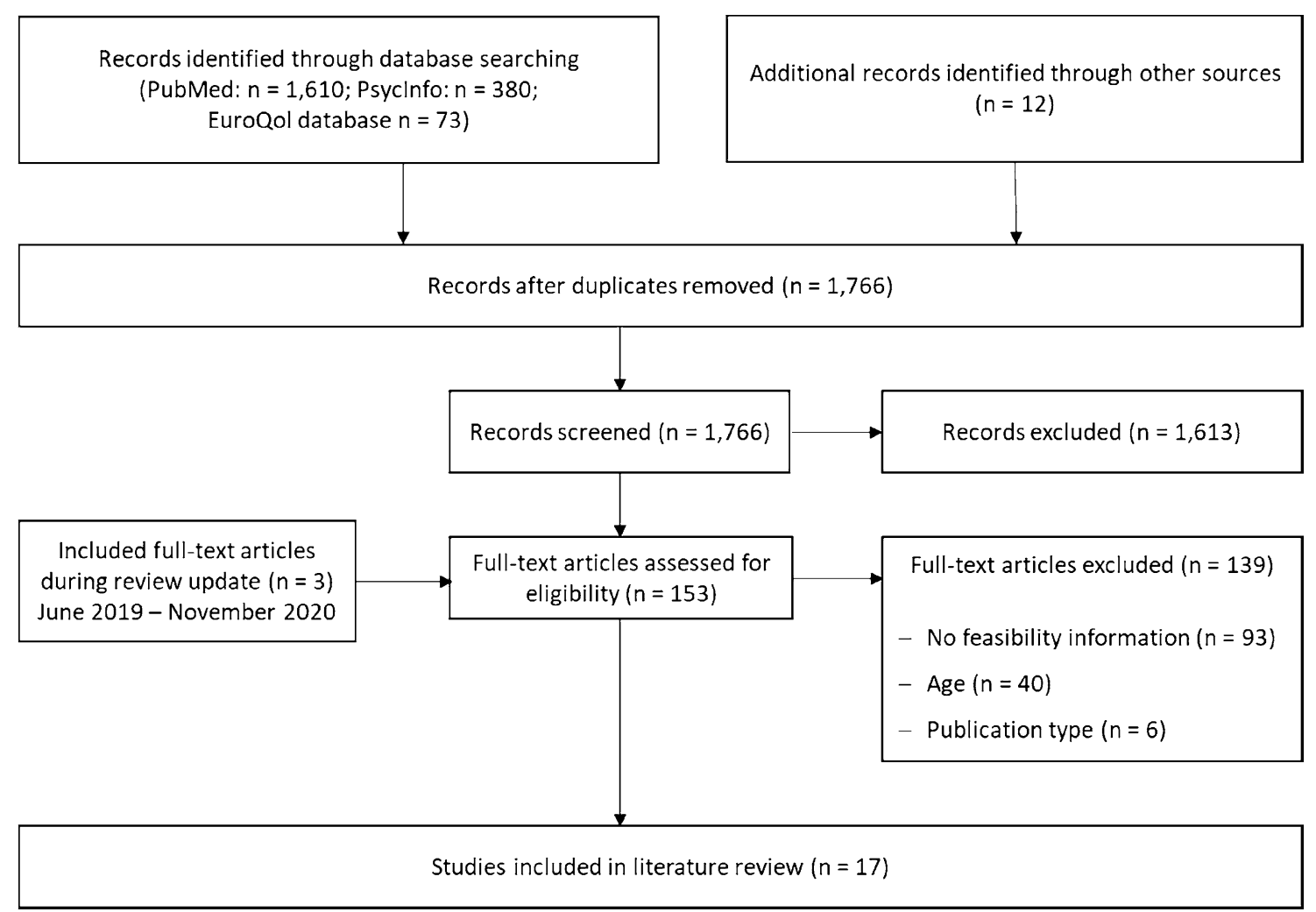

Fig. 1 Flow chart of the literature search and study selection process 


\section{Study characteristics}

Studies were mostly conducted in Europe except three studies from Australia, Canada and South Africa (Table 1). The reported sample size ranges from 10 to 3073 respondents with data being collected in both general $(n=4)$ and patient populations $(n=13)$. One study applied a qualitative approach to assess the feasibility of the EQ-5D-3L in older adults, whereas the remaining 16 studies used a quantitative study design (Table 2). Only two of the included studies used the EQ-5D-5L, whereas the 3L was used in the remaining 14 studies, with one study additionally using a cognitive bolton. The use of the EQ VAS is inconsistent; two studies make no statements towards its use, whilst one study explicitly states that the EQ VAS was not applied. The remaining 14 studies provide information based on the EQ VAS.

With regard to the administration mode two studies did not make any specific statements as to how the EQ-5D was administered (Table 2). The majority of studies $(n=13)$ applied a self-complete version of the EQ-5D to collect HRQoL data, where interviewer support was available on request in nine of those studies. Moreover, two of the included studies applied an interviewer-based approach to collect EQ-5D data in the elderly population.

\section{Feasibility information}

We included 17 studies examining aspects of the EQ-5D's feasibility in the elderly. However, only eight studies referred to this topic as "feasibility", whilst another seven studies referred to this under the term "completion" and one further study each investigated these properties labelled as "practicality" or "acceptability". Included studies assessed the EQ-5D's feasibility in terms of missing values $(n=11)$, completion rates $(n=13)$, made qualitative statements towards the completion $(n=9)$ and measured the time required to complete the EQ-5D $(n=3)$. One study investigated all four aspects, whilst four studies reported results on three of these aspects; six studies examined at least two feasibility outcomes, whilst six studies only reported on one of the feasibility aspects (Table 2).

Detailed information on missing data for the EQ-5D-3L was reported by five studies, whilst three additional studies only report that missing values did not exceed $10 \%$ in general. At the dimension-level, the proportion of missing values did not exceed $10.7 \%$ across all five dimensions. In addition, information on missing values for the $5 \mathrm{~L}$ descriptive system was only provided by Grund et al. [40], where only responses to the usual activities dimension were missing in $10.5 \%$ of the cases. Missing values on the EQ VAS were evaluated in nine studies. Three studies found no missing values and one study reported that less than $10 \%$ were missing. Further, five studies reported the specific proportion of missing responses to the EQ VAS ranging from 2.3 up to $25.3 \%$, generally exceeding the share of missing values on the descriptive system.

Completion of the EQ-5D-3L was either around but mostly above $90 \%$ for the baseline assessment, except in two studies which reported a completion of just above $80 \%$. Luthy et al. [41] provided age-specific completion rates of $94 \%$ for the total sample, still achieving above $90 \%$ for respondents aged 90 years and above. Again, only Grund et al. [40] reported a completion rate of $89.5 \%$ for the EQ-5D-5L.

Completion time was assessed in three studies. Both the EQ-5D-3L and EQ-5D-5L can usually be completed in less than five minutes (more details in Table 2) [40, 42, 43]. As can be assumed, respondents self-completing the EQ-5D need less time to complete than respondents who are in need of assistance, but were found to need similar amounts of time than respondents with a strong need for assistance, essentially administering the EQ-5D-5L in an interviewerbased approach [40].

Moreover, nine studies described the completion of the EQ-5D qualitatively. Overall, the measures were found to be brief and easy to use [20,40,44, 45]. Comprehension issues were rarely reported, but related to narrow item interpretation or restrictive item wording, where only few respondents had problems mapping their response to the descriptive system [42, 44, 46, 47]. Moreover, comprehension issues with regard to the EQ VAS were also reported in three studies [43, 46, 47], where Hulme et al. [46] stated that $27 \%$ of respondents had trouble completing or understanding the EQ VAS.

Coast et al. [48] assessed whether respondents required an interviewer-based approach controlling for age. In total, 50\% of their sample required an interviewer to complete the EQ5D-3L; whilst stratifying for age the probability of requiring interviewer administration was at $11 \%$ at 65 years, $37 \%$ at 75 years and at $73 \%$ at age 85 . At a similar level Hulme et al. [46] found that 55\% required additional help from an interviewer whilst answering the EQ-5D-3L.

\section{Discussion}

Over the past three decades the EQ-5D has been applied in an extensive list of populations and settings and amongst those elderly populations and patients were frequently examined, too. Even though the EQ-5D's feasibility properties were confirmed and found to be unproblematic for the overall general population by two recent major reviews [22, 23], feasibility of the EQ-5D is not systematically explored for the elderly population and warrants further examination $[18,49]$. Therefore, this review summarised the available information on the feasibility properties of 


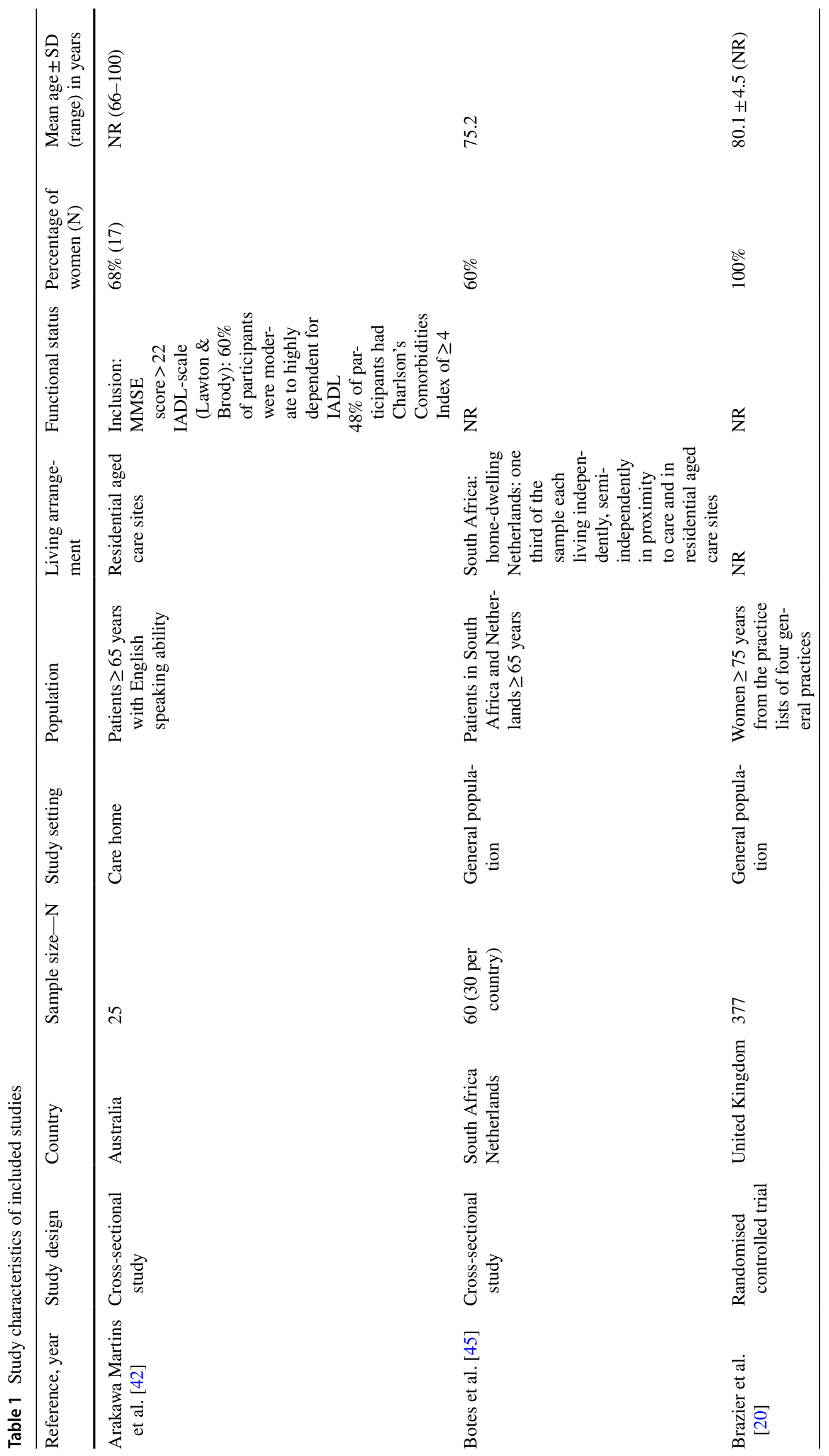




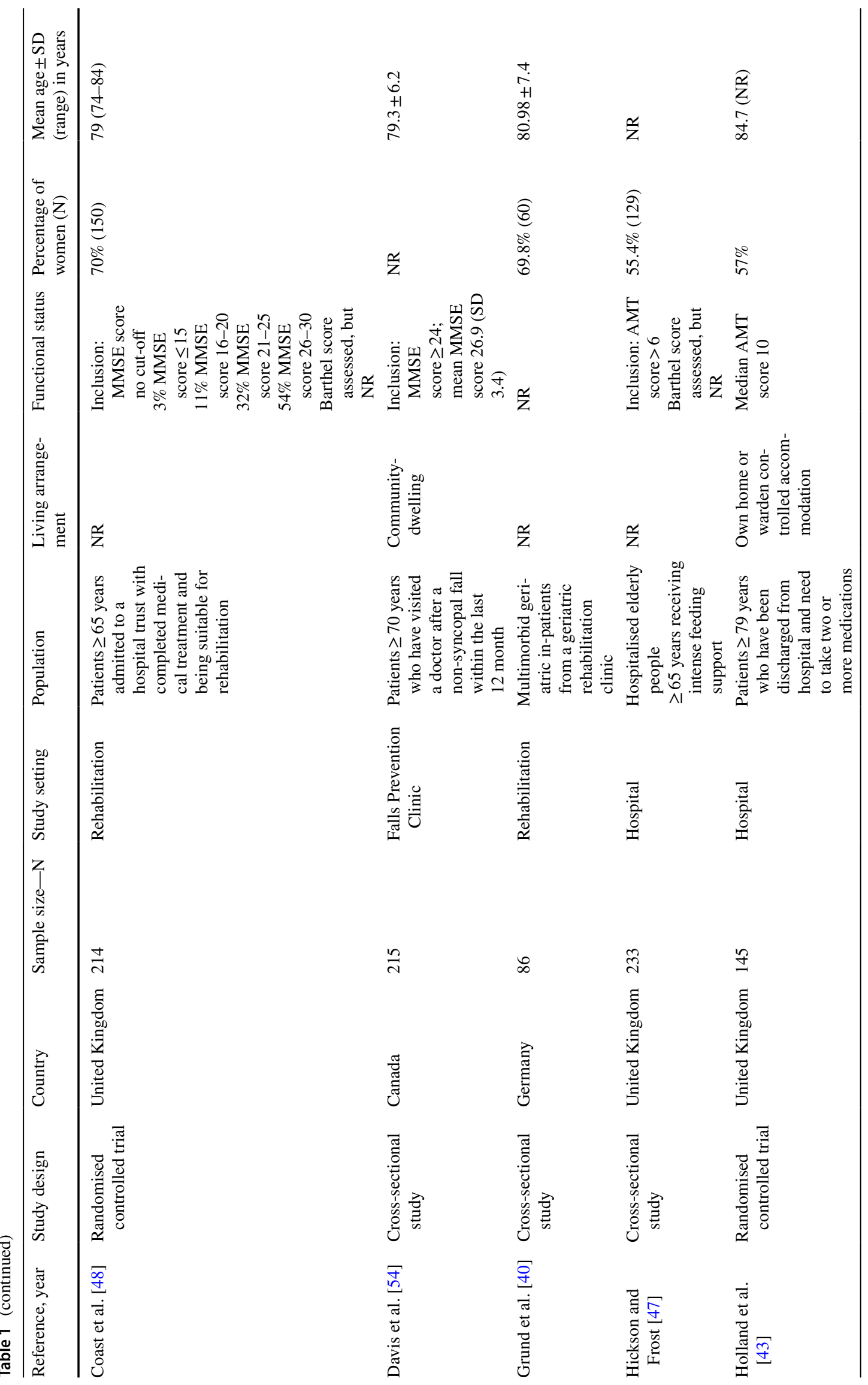




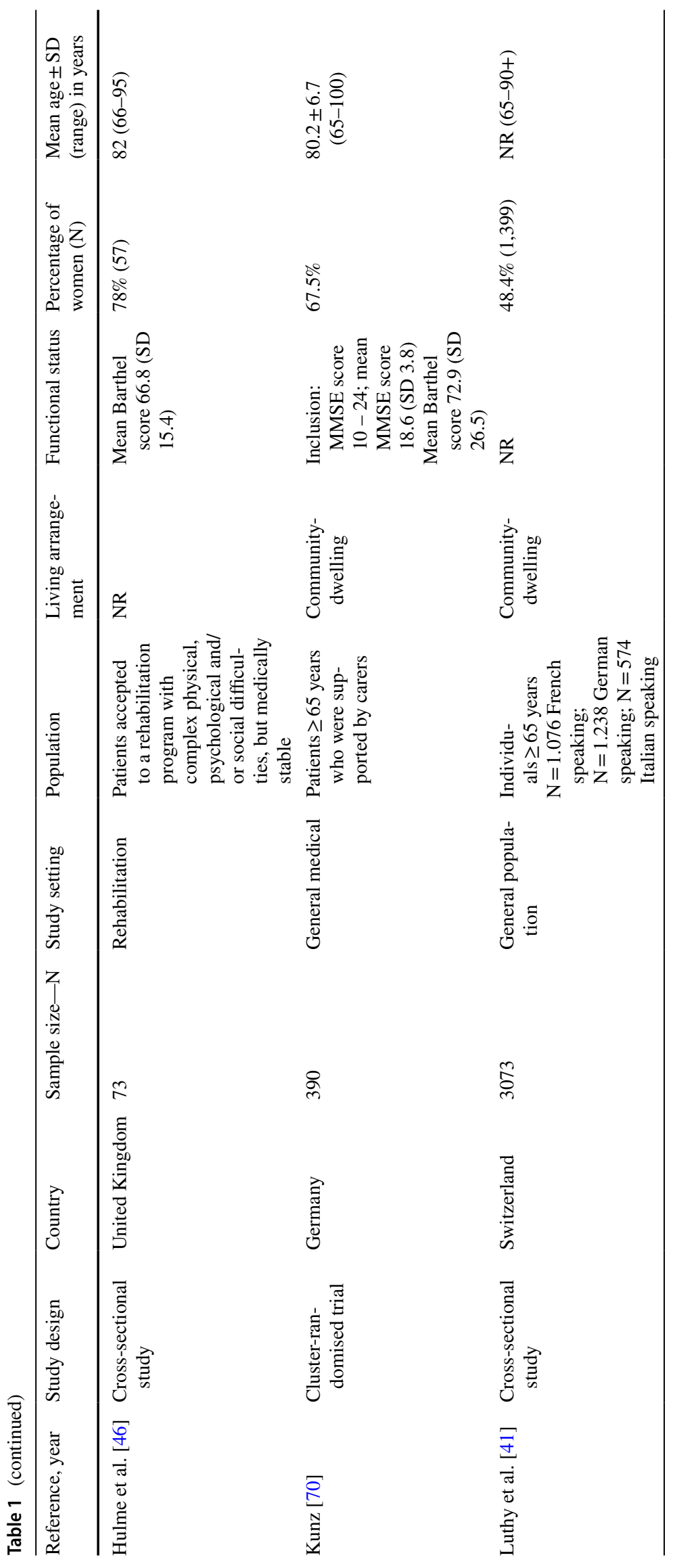




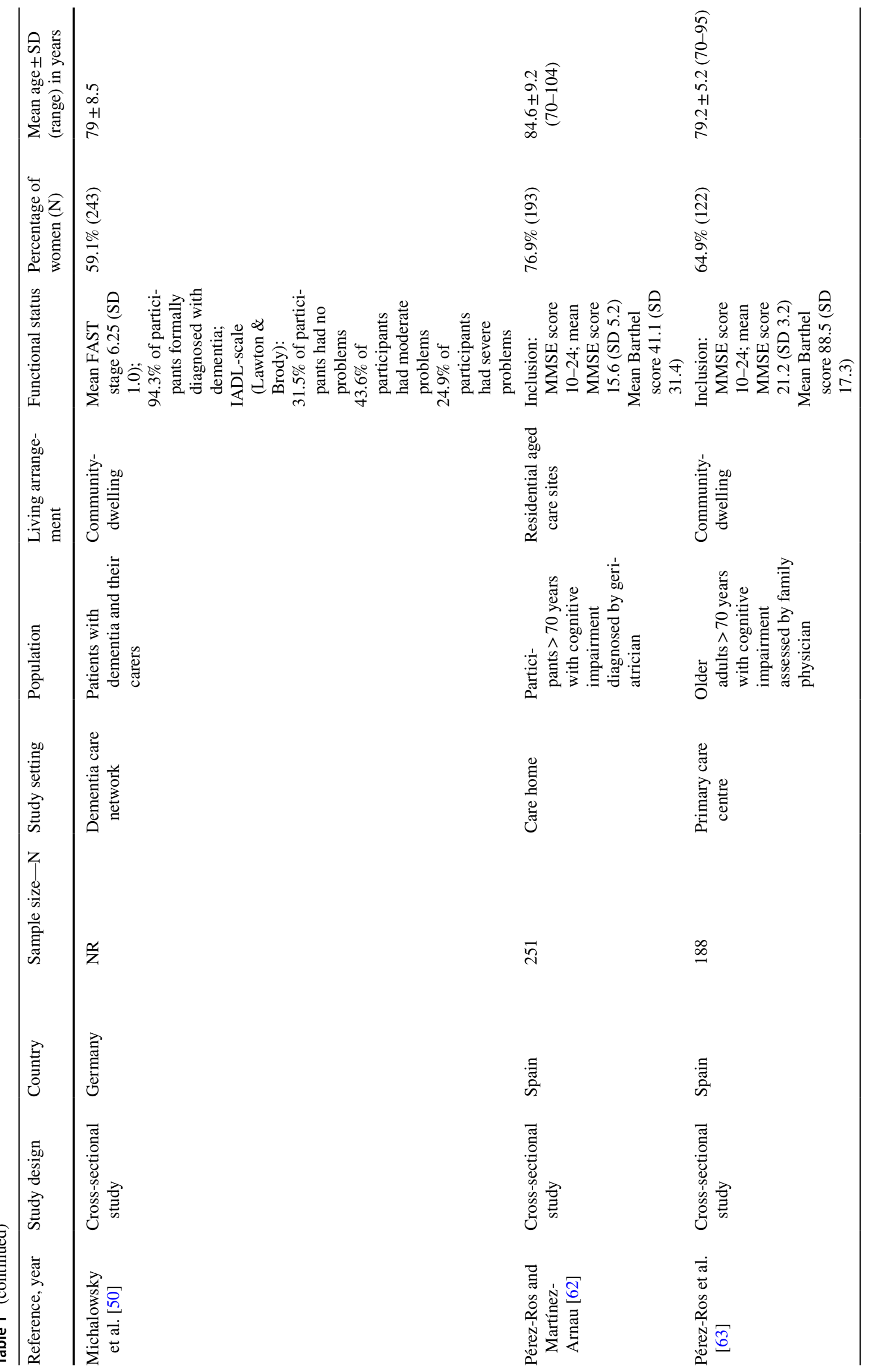




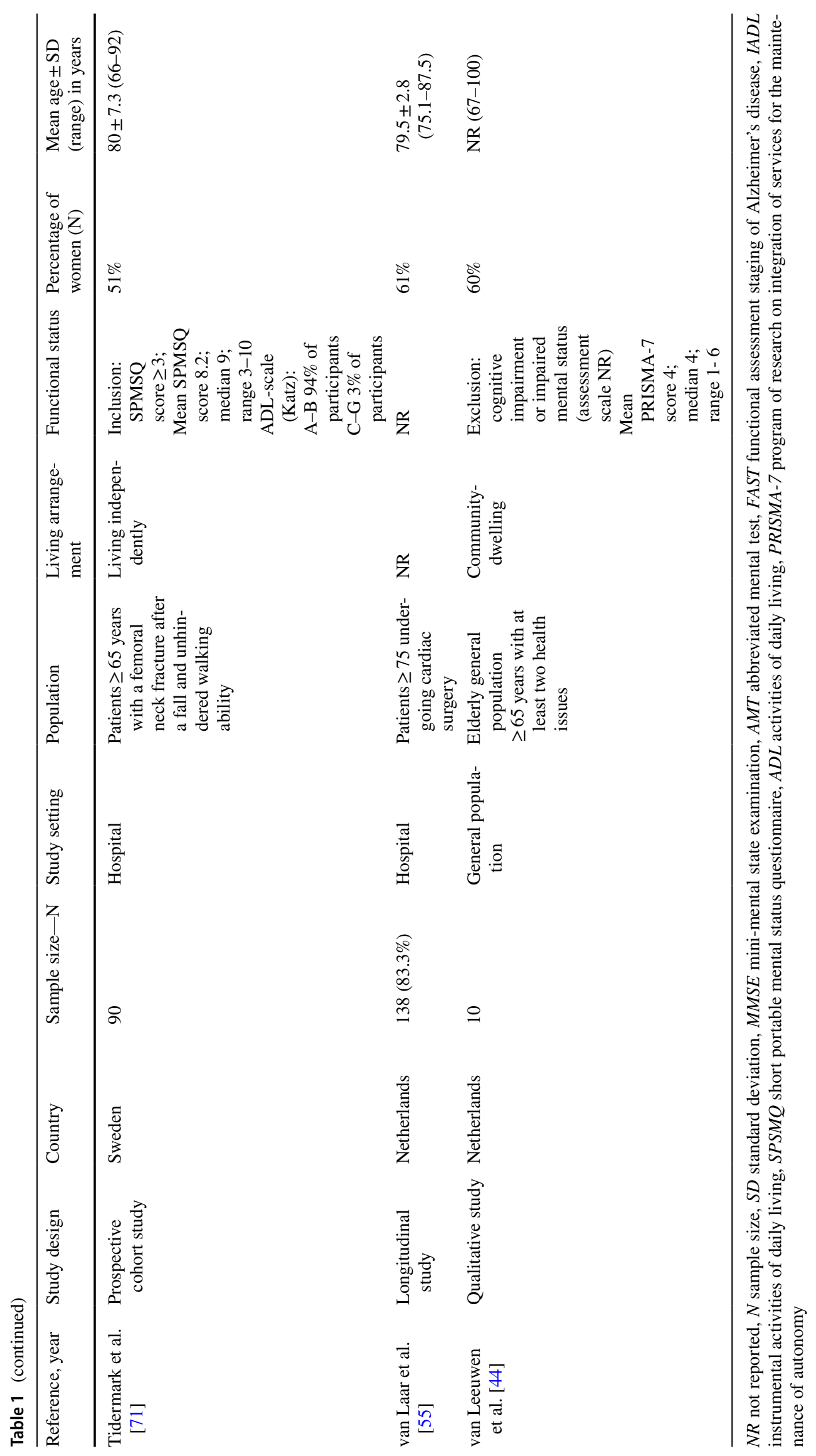




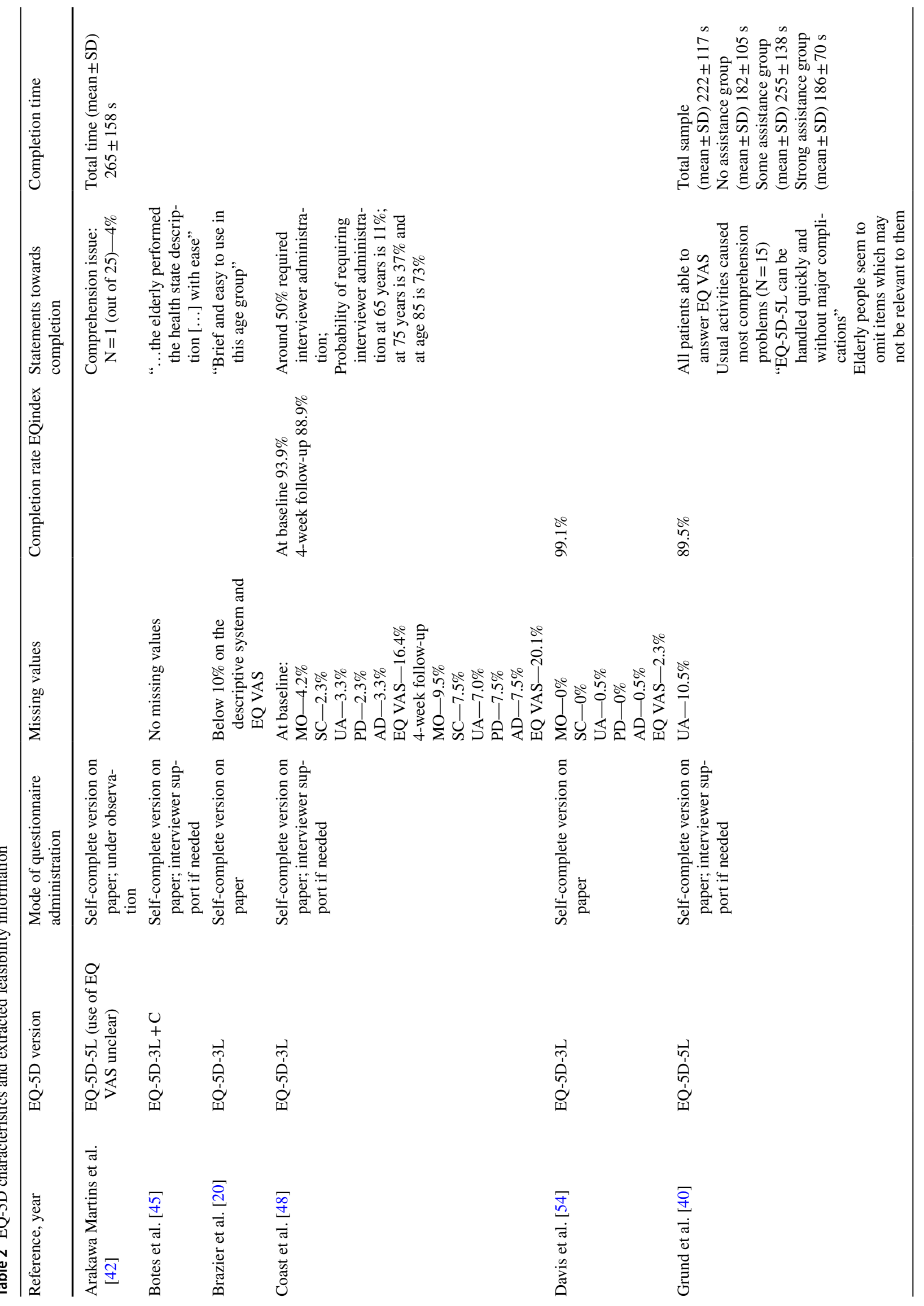




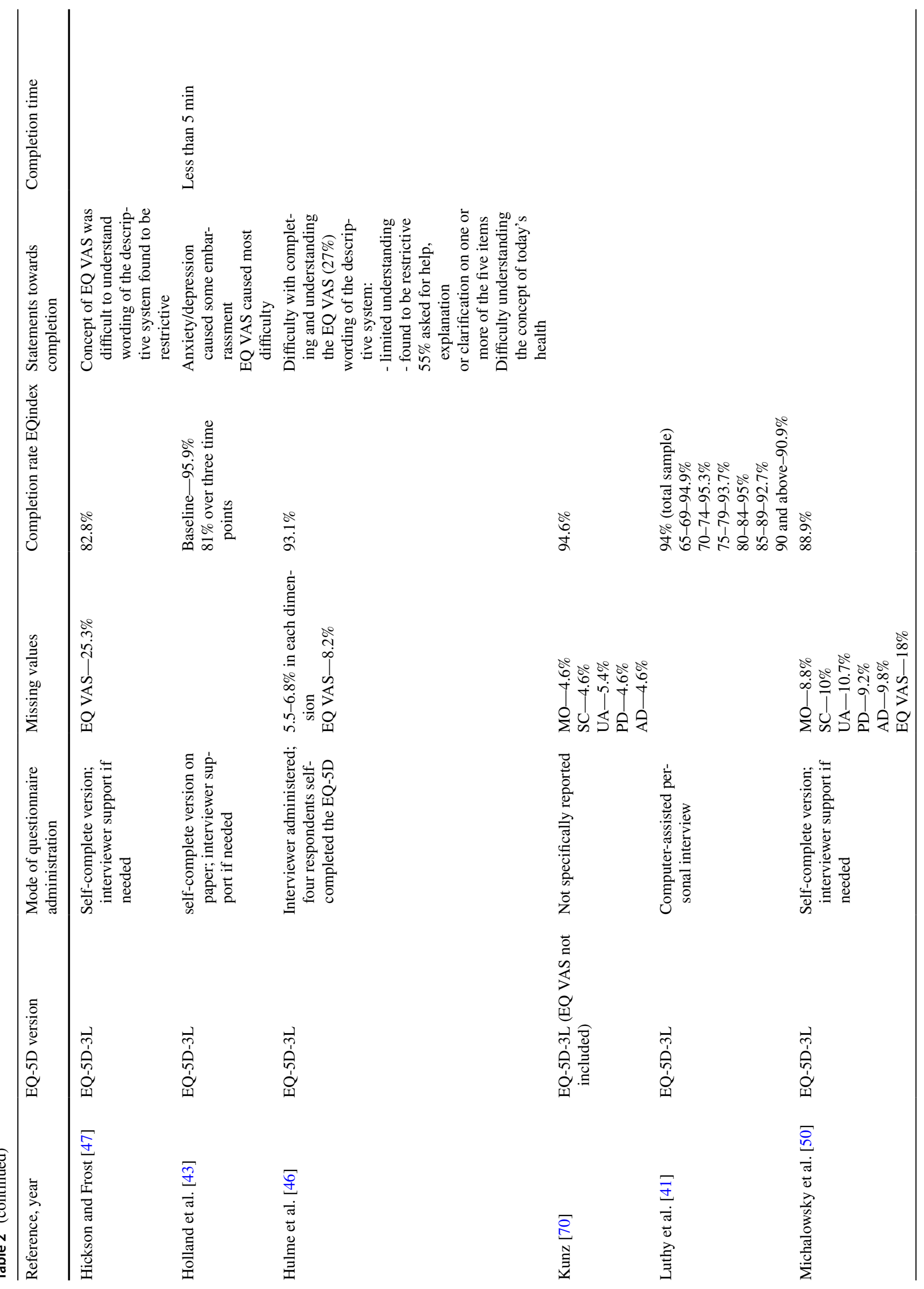




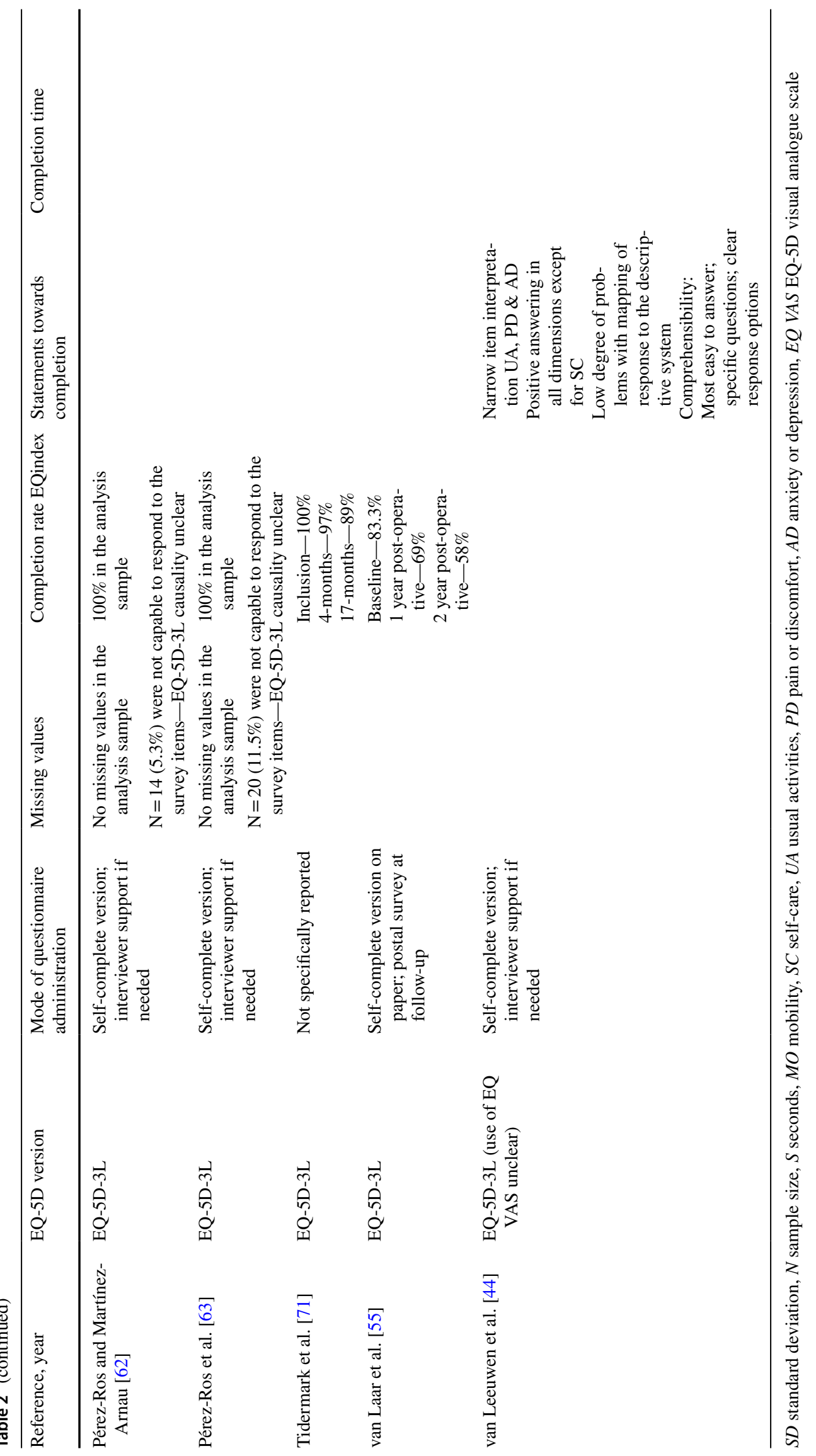


both the EQ-5D-3L and EQ-5D-5L in the elderly population. In the light of the reviewed studies, the terminology around this measurement property and which aspects of feasibility are commonly reported are inconsistently used. Overall, missing values of approximately up to $10 \%$ on the descriptive system and completion rates of around $90 \%$ seem to be ballpark figures in elderly populations. On the other hand, completion of the EQ VAS seems to pose a higher burden to older respondents, since missing rates are generally higher. Also of interest is the high prevalence of interviewer-assisted or even interviewer-based administration of the EQ-5D in the older population.

This review identified several different synonyms for feasibility such as 'completion', 'practicality' or 'acceptability'. However, all labels aim to describe the applicability of the measure to the target population in a similar manner, viz. how conveniently and successfully the measure can be completed. Yet, to the best of our knowledge, none of the leading QoL organisations such as the EuroQol group, the international society for quality of life research (ISOQOL), World Health Organization (WHO) or the European Organisation for Research and Treatment of Cancer (EORTC) Quality of Life Group provide a definition of feasibility. To aid the standardisation of reporting this measurement property and the usability of available evidence for researchers, we suggest summarising these labels under the term 'feasibility'.

Regardless of the label used in individual studies, there was little agreement on how feasibility was operationalised and therefore included studies examined feasibility in terms of missing values, completion rate, time required to complete the EQ-5D or-more broadly-in qualitative statements referring to the completion of the instrument. However, the amount of available information on each of these parameters varied greatly. One of the more frequently reported outcomes were missing values. Overall, the proportion of missing values was mostly below $7 \%$ on the five dimensions with few exceptions, e.g. in a study by Michalowsky et al. [50] including cognitively impaired respondents; but generally missing values did not exceed $11 \%$. Where detailed information on the dimension-level was available, those suggested that dimensions were equally affected with no particular one sticking out. Two recent systematic reviews provide a reference value of $5 \%$ missing values for both the EQ-5D-3L and EQ-5D-5L $[22,23]$. This upper value largely seems to hold for the included studies with only a marginal excess effect in missing values in the elderly population, which was already described elsewhere [30, 51-53]. Even though the proportion of missing values seems to increase slightly with higher age, the EQ-5D compared more favourably in terms of missing values when compared to other instruments that are commonly applied with the elderly such as the SF-36 [20, 40], the AQoL [43] or ICECAP-O [54].
A related concept is the completion rate of the descriptive system. Findings from this review suggest that the proportion of incomplete responses is commonly less than $10 \%$, i.e. more than $90 \%$ of index values are computable. Considerably lower completion rates were reported by Hickson and Frost [47], arguing that patients were too ill to complete the questionnaire. Also, van Laar et al. [55] report an equally low completion rate of $83.3 \%$, however, it remains unclear to what extent the study design or the disease negatively interfered with completion rates. Again, comparing our findings with the benchmark completion rates of more than 93.4\% (3L) and 96\% (5L) provided by Buchholz et al. [22], we find that the share of computable index values seems to slightly decrease in the elderly population. This is congruent with findings from Luthy et al. [41] who found that completion rates for the EQ-5D-3L were negatively associated with increasing age. However, this finding is not surprising given that missing values were also slightly more prevalent in the elderly, which in turn lead to incomplete health state information. Based on the reviewed evidence on missing values and completion rates of the descriptive system, we would like to argue that a magnitude of approximately less than $10 \%$ missing values and about $90 \%$ of computable EQ-5D index values demonstrate reasonable feasibility of the EQ-5D in the elderly population.

A further component that was found to be more frequently missing was the EQ VAS. The range of missing EQ VAS ratings was $2.3-25.3 \%$ and differs considerably, but generally exceeds the proportion of missing values in the descriptive system. There is evidence that the concept of the EQ VAS is more difficult to comprehend than the descriptive system and causes the most problems [43, 46-48], which was found in older populations before [26]. Then again, higher proportions of missing EQ VAS ratings [32] and execution problems [56] were also reported for the younger populations, yet to a lesser extent.

Generally, missing data on both components the EQ VAS and the descriptive system diminish the available sample size for analyses and, if item nonresponse occurs systematically, this may result in biased results [57]. The decision on how to handle missing data in the analysis should be guided and justified based on the mechanism of missing data rather than the proportion of missing values per se [21,39, 58-60]. Generally, preventing missing data before they occur is more efficient than an analytical remedy [61]. In this sense, a reoccurring topic in included studies is the high proportion of older respondents requiring assistance to complete the EQ-5D or even an interviewer-based approach [40, 46, 48]. Several issues, such as asking for explanations and clarifications due to limited item interpretation [40, 46, 48], were identified that may warrant interviewer support when collecting EQ-5D data in the elderly [44]. Similar findings were presented from a qualitative study with younger hip fracture 
patients (60+ years) by Rohr et al. [37] arguing that without interviewer support missing values would be significantly higher. At least three of the included studies acknowledged that interviewer support was needed, but did not further assess the amount of support that was required by the participants [47, 62, 63]. With regard to this, Coast et al. [48] report age-dependent probabilities of requiring interviewer assistance, further suggesting an increased need of assistance with higher age. On the other hand, several studies report good feasibility properties in the older population relying on self-completed measures without assistance [20, 42, 64], whereby the EQ-5D appears to be well applicable as a selfreport measure in the elderly. Even though interviewer support was frequently provided in included studies, the effect of interviewer assistance on preventing missing values on the descriptive system and EQ VAS or increased completion rates cannot be quantified based on the available evidence for two reasons. First, included studies did not compare feasibility aspects between assisted and non-assisted respondent sub-groups and, secondly, studies were too heterogeneous in their outcomes and study characteristics to identify factors that facilitate adequate feasibility. Furthermore, adopting an interview approach is resource intensive and may introduce additional bias or measurement error, if implemented improperly and, thus, may offset potential benefits [65]. The controversial evidence and the lack of studies exploring the effect of an interviewer approach express the need for further research on this topic. Future qualitative research as done by Rohr et al. [37] and van Leeuwen et al. [44] may help to better understand the role of interviewers in the data collection process. Alternatively, a cognitive de-briefing of interviewers in quantitative studies may shed a light on the heterogeneity of the elderly and help exploring sub-groups that might benefit from interviewer assistance.

From our review, we found only two of the included studies applied the EQ-5D-5L in the elderly population and analysed aspects of the instrument's feasibility, which limits the generalisability of our findings beyond these two studies for the EQ-5D-5L. However, we would expect the EQ-5D-5L's feasibility to be comparable or potentially even better than those of the EQ-5D-3L, since the EQ-5D-5L kept the brevity. Further, enabling respondents to better map their health onto the descriptive system in more detailed distinctions might potentially increase engagement to the task [66]. But then again, this may have also increased the cognitive burden hampering instrument completion and data quality [67-69], however, not to an extent which is believed to overburden respondents [66]. Since none of the included studies comparatively examined both the EQ-5D-3L and EQ-5D-5L in the elderly population, this gap warrants further research.

Some limitations of this study should be considered. In accordance with the underlying age definition for the elderly population, this study only included publications, which focussed on study populations exclusively being 65 years and above. Due to this strict age-related eligibility criterion, some studies were excluded where the majority of respondents may have met the inclusion criteria, whilst only a minor share of respondents was not eligible. Also, included studies exclusively sampled respondents from western and developed countries, which does not allow any generalisation on the feasibility in elderly populations in Asia, Africa or South America, where the older population may be assumed to be different due to their cultural believes or lower literacy in rural regions. Furthermore, the heterogeneity of the target population, which may include healthy and independently living respondents, but also frail or cognitively impaired participants, also limits the generalisability of our findings, especially with regard to the EQ-5D-5L, which was only included in two studies. Moreover, despite searching several databases, we might have missed relevant publications due to an inconsistent use of terminology in addressing feasibility and more importantly due to different search engines for the databases, which may have partially prevented the identification of studies addressing feasibility as a secondary or even only as a descriptive analysis. As a potential remedy to this limitation, we searched all reference lists of included articles to mitigate this risk.

\section{Conclusion}

This study aimed to assess the EQ-5D's feasibility properties when used in the elderly population. Our findings suggest that missing values or comprehension problemsespecially with the EQ VAS - are slightly more prevalent in older age groups compared to the younger general population. However, these aspects are well within an acceptable range and still considerably lower than in other measures such as SF-36 or ICECAP-O, which are frequently used in the elderly. Furthermore, older respondents seem to have a higher propensity of requiring some degree of assistance or even an interviewer-based approach. Overall, evidence from the reviewed literature indicates that the EQ-5D-3L has good feasibility properties and, hence, is highly applicable in older respondents. Moreover, further research is needed to explore feasibility properties of the EQ-5D-5L in older respondents too, whilst examining the proportion of missing values, completion rate and completion time considering the role of any interviewer support in the data collection process.

Funding Open Access funding enabled and organized by Projekt DEAL. This research was funded by the EuroQol Research Foundation under Grant No. 2015200. The views expressed by the authors in the publication do not necessarily reflect the view of the EuroQol Group. 


\section{Declarations}

Conflict of interest Ole Marten and Wolfgang Greiner are members of the EuroQol Group. There are no other conflict of interest.

Ethical approval This is a review paper and therefore none of the authors conducted human or animal data collection.

Open Access This article is licensed under a Creative Commons Attribution 4.0 International License, which permits use, sharing, adaptation, distribution and reproduction in any medium or format, as long as you give appropriate credit to the original author(s) and the source, provide a link to the Creative Commons licence, and indicate if changes were made. The images or other third party material in this article are included in the article's Creative Commons licence, unless indicated otherwise in a credit line to the material. If material is not included in the article's Creative Commons licence and your intended use is not permitted by statutory regulation or exceeds the permitted use, you will need to obtain permission directly from the copyright holder. To view a copy of this licence, visit http://creativecommons.org/licenses/by/4.0/.

\section{References}

1. OECD. (2021). Elderly population (indicator). OECD Publishing.

2. United Nations (UN). (2019). World population prospects. United Nations.

3. Kingston, A., Robinson, L., Booth, H., Knapp, M., \& Jagger, C. (2018). Projections of multi-morbidity in the older population in England to 2035: Estimates from the population ageing and care simulation (PACSim) model. Age and Ageing, 47(3), 374-380. https://doi.org/10.1093/ageing/afx201

4. Salive, M. E. (2013). Multimorbidity in older adults. Epidemiologic Reviews, 35(1), 75-83. https://doi.org/10.1093/epirev/ mxs009

5. Wolff, J. L., Starfield, B., \& Anderson, G. (2002). Prevalence, expenditures, and complications of multiple chronic conditions in the elderly. Archives of Internal Medicine, 162(20), 2269-2276. https://doi.org/10.1001/archinte.162.20.2269

6. Drummond, M. F., Sculpher, M. J., Claxton, K., Stoddart, G. L., \& Torrance, G. W. (2015). Methods for the economic evaluation of health care programmes (4th ed.). Oxford University Press.

7. Devlin, N. J., \& Brooks, R. (2017). EQ-5D and the EuroQol group: Past, present and future. Applied Health Economics and Health Policy, 15(2), 127-137. https://doi.org/10.1007/ s40258-017-0310-5

8. Brooks, R. (1996). EuroQol: The current state of play. Health Policy, 37(1), 53-72. https://doi.org/10.1016/0168-8510(96)00822-6

9. Devlin, N., Parkin, D., \& Janssen, B. (2020). Methods for analysing and reporting EQ-5D data (1st ed.). Springer.

10. Herdman, M., Gudex, C., Lloyd, A., Janssen, M., Kind, P., Parkin, D., Bonsel, G., \& Badia, X. (2011). Development and preliminary testing of the new five-level version of EQ-5D (EQ-5D-5L). Quality of Life Research, 20(10), 1727-1736. https://doi.org/10.1007/ s11136-011-9903-x

11. EuroQol Research Foundation (2019). EQ-5D-5L user guide

12. EuroQol Research Foundation (2018). EQ-5D-3L user guide

13. Janssen, M. F., Pickard, A. S., Golicki, D., Gudex, C., Niewada, M., Scalone, L., Swinburn, P., \& Busschbach, J. (2013). Measurement properties of the EQ-5D-5L compared to the EQ5D-3L across eight patient groups: a multi-country study. Quality of Life Research, 22(7), 1717-1727. https://doi.org/10.1007/ s11136-012-0322-4
14. Wisløff, T., Hagen, G., Hamidi, V., Movik, E., Klemp, M., \& Olsen, J. A. (2014). Estimating QALY gains in applied studies: A review of cost-utility analyses published in 2010. PharmacoEconomics, 32(4), 367-375. https://doi.org/10.1007/ s40273-014-0136-Z

15. Bulamu, N. B., Kaambwa, B., \& Ratcliffe, J. (2015). A systematic review of instruments for measuring outcomes in economic evaluation within aged care. Health and Quality of Life Outcomes, 13, 179. https://doi.org/10.1186/s12955-015-0372-8

16. Makai, P., Brouwer, W. B. F., Koopmanschap, M. A., Stolk, E. A., \& Nieboer, A. P. (2014). Quality of life instruments for economic evaluations in health and social care for older people: A systematic review. Social Science \& Medicine, 1982(102), 83-93. https:// doi.org/10.1016/j.socscimed.2013.11.050

17. Hickey, A., Barker, M., McGee, H., \& O’Boyle, C. (2005). Measuring health-related quality of life in older patient populations: A review of current approaches. PharmacoEconomics, 23(10), 971-993. https://doi.org/10.2165/00019053-20052 3100-00002

18. Haywood, K. L., Garratt, A. M., \& Fitzpatrick, R. (2005). Quality of life in older people: A structured review of generic selfassessed health instruments. Quality of Life Research, 14(7), 1651-1668. https://doi.org/10.1007/s11136-005-1743-0

19. Cleland, J., Hutchinson, C., Khadka, J., Milte, R., \& Ratcliffe, J. (2019). A review of the development and application of generic preference-based instruments with the older population. Applied Health Economics and Health Policy, 17(6), 781-801. https://doi. org/10.1007/s40258-019-00512-4

20. Brazier, J. E., Walters, S. J., Nicholl, J. P., \& Kohler, B. (1996). Using the SF-36 and Euroqol on an elderly population. Quality of Life Research, 5(2), 195-204. https://doi.org/10.1007/bf00434741

21. Morris, S., Devlin, N. J., Parkin, D., \& Spencer, A. (2012). Economic analysis in health care ( 2 nd ed.). Wiley.

22. Buchholz, I., Janssen, M. F., Kohlmann, T., \& Feng, Y.-S. (2018). A systematic review of studies comparing the measurement properties of the three-level and five-level versions of the EQ-5D. PharmacoEconomics, 36(6), 645-661. https://doi.org/10.1007/ s40273-018-0642-5

23. Feng, Y.-S., Kohlmann, T., Janssen, M. F., \& Buchholz, I. (2021). Psychometric properties of the EQ-5D-5L: A systematic review of the literature. Quality of Life Research, 30(3), 647-673. https:// doi.org/10.1007/s11136-020-02688-y

24. Lutomski, J. E., Krabbe, P. F. M., Bleijenberg, N., Blom, J., Kempen, G. I. J. M., MacNeil-Vroomen, J., Muntinga, M. E., Steyerburg, E., Olde-Rikkert, M. G. M., \& Melis, R. J. F. (2017). Measurement properties of the EQ-5D across four major geriatric conditions: Findings from TOPICS-MDS. Health and Quality of Life Outcomes, 15(1), 45. https://doi.org/10.1186/ s12955-017-0616-x

25. van Leeuwen, K. M., Bosmans, J. E., Jansen, A. P. D., Hoogendijk, E. O., van Tulder, M. W., van der Horst, H. E., \& Ostelo, R. W. (2015). Comparing measurement properties of the EQ-5D-3L, ICECAP-O, and ASCOT in frail older adults. Value in Health, 18(1), 35-43. https://doi.org/10.1016/j.jval.2014.09.006

26. Streiner, D. L., Norman, G. R., \& Cairney, J. (2015). Health measurement scales: A practical guide to their development and use (5th ed.). Oxford University Press.

27. Prinsen, C. A. C., Mokkink, L. B., Bouter, L. M., Alonso, J., Patrick, D. L., de Vet, H. C. W., \& Terwee, C. B. (2018). COSMIN guideline for systematic reviews of patient-reported outcome measures. Quality of Life Research, 27(5), 1147-1157. https://doi. org/10.1007/s11136-018-1798-3

28. Golicki, D., Niewada, M., Buczek, J., Karlińska, A., Kobayashi, A., Janssen, M. F., \& Pickard, A. S. (2015). Validity of EQ-5D-5L in stroke. Quality of Life Research, 24(4), 845-850. https://doi. org/10.1007/s11136-014-0834-1 
29. Agborsangaya, C. B., Lahtinen, M., Cooke, T., \& Johnson, J. A. (2014). Comparing the EQ-5D 3L and 5L: Measurement properties and association with chronic conditions and multimorbidity in the general population. Health and Quality of Life Outcomes, 12, 74. https://doi.org/10.1186/1477-7525-12-74

30. Gerard, K., Nicholson, T., Mullee, M., Mehta, R., \& Roderick, P. (2004). EQ-5D versus SF-6D in an older, chronically Ill patient group. Applied Health Economics and Health Policy, 3(2), 91-102. https://doi.org/10.2165/00148365-200403020-00005

31. Yfantopoulos, J., Chantzaras, A., \& Kontodimas, S. (2017). Assessment of the psychometric properties of the EQ-5D-3L and EQ-5D-5L instruments in psoriasis. Archives of Dermatological Research, 309(5), 357-370. https://doi.org/10.1007/ s00403-017-1743-2

32. Badia, X., Schiaffino, A., Alonso, J., \& Herdman, M. (1998). Using the EuroQoI 5-D in the Catalan general population: Feasibility and construct validity. Quality of Life Research, 7(4), 311-322. https://doi.org/10.1023/a:1024933913698

33. Halvorsrud, L., \& Kalfoss, M. (2014). Quality of life data in older adults: self-assessment vs interview. British Journal of Nursing, 23(13), 712-721. https://doi.org/10.12968/bjon.2014.23.13.712

34. Weichbold, M., \& Kutschar, P. (2019). Interviewing elderly in nursing homes-respondent and survey characteristics as predictors of item nonresponse. Survey Methods, 2019, 1-9.

35. Bowling, A. (2005). Mode of questionnaire administration can have serious effects on data quality. Journal of Public Health, 27(3), 281-291. https://doi.org/10.1093/pubmed/fdi031

36. Hays, R. D., Kim, S., Spritzer, K. L., Kaplan, R. M., Tally, S., Feeny, D., Liu, H., \& Fryback, D. G. (2009). Effects of mode and order of administration on generic health-related quality of life scores. Value in Health, 12(6), 1035-1039. https://doi.org/10. 1111/j.1524-4733.2009.00566.x

37. Rohr, M., Brandstetter, S., Plomer, A.-S., Loss, J., Kretschmer, R., \& Apfelbacher, C. (2021). A qualitative study exploring content validity and feasibility of frequently used generic health-related quality of life measures in older people with hip fracture: The patients' perspective. Injury, 52(2), 134-141. https://doi.org/10. 1016/j.injury.2020.09.061

38. EuroQol Research Foundation. EuroQol website

39. Simons, C. L., Rivero-Arias, O., Yu, L.-M., \& Simon, J. (2015). Multiple imputation to deal with missing EQ-5D-3L data: Should we impute individual domains or the actual index? Quality of Life Research, 24(4), 805-815. https://doi.org/10.1007/ s11136-014-0837-y

40. Grund, S., Breitinger, E., Fricke, S., Alpers, G. W., Hundsdörfer, W., \& Schafer, H. G. (2017). Health-related quality of life measurement inpatient geriatric rehabilitation: A comparison of the feasibility and suitability between the SF-36 and EQ-5D-5L questionnaires. Journal of Gerontology \& Geriatric Research. https://doi.org/10.4172/2167-7182.1000458

41. Luthy, C., Cedraschi, C., Allaz, A.-F., Herrmann, F. R., \& Ludwig, C. (2015). Health status and quality of life: Results from a national survey in a community-dwelling sample of elderly people. Quality of Life Research, 24(7), 1687-1696. https://doi.org/10.1007/ s11136-014-0894-2

42. Arakawa Martins, B., Barrie, H., Dollard, J., Mahajan, N., \& Visvanathan, R. (2018). Older adults' perceptions of the built environment and associations with frailty: A feasibility and acceptability study. The Journal of Frailty \& Aging, 7(4), 268-271. https:// doi.org/10.14283/jfa.2018.23

43. Holland, R., Smith, R. D., Harvey, I., Swift, L., \& Lenaghan, E. (2004). Assessing quality of life in the elderly: A direct comparison of the EQ-5D and AQoL. Health Economics, 13(8), 793-805. https://doi.org/10.1002/hec.858

44. van Leeuwen, K. M., Jansen, A. P. D., Muntinga, M. E., Bosmans, J. E., Westerman, M. J., van Tulder, M. W., \& van der Horst,
H. E. (2015). Exploration of the content validity and feasibility of the EQ-5D-3L, ICECAP-O and ASCOT in older adults. BMC Health Services Research, 15, 201. https://doi.org/10.1186/ s12913-015-0862-8

45. Botes, R., Vermeulen, K. M., Ranchor, A. V., \& Buskens, E. (2018). Functional health state description and valuation by people aged 65 and over: A pilot study. BMC Geriatrics, 18(1), 11. https://doi.org/10.1186/s12877-018-0711-9

46. Hulme, C., Long, A. F., Kneafsey, R., \& Reid, G. (2004). Using the EQ-5D to assess health-related quality of life in older people. Age and Ageing, 33(5), 504-507. https://doi.org/10.1093/ageing/ afh 178

47. Hickson, M., \& Frost, G. (2004). An investigation into the relationships between quality of life, nutritional status and physical function. Clinical Nutrition, 23(2), 213-221. https://doi.org/10. 1016/S0261-5614(03)00127-4

48. Coast, J., Peters, T. J., Richards, S. H., \& Gunnell, D. J. (1998). Use of the EuroQoL among elderly acute care patients. Quality of Life Research, 7(1), 1-10. https://doi.org/10.1023/a:1008857203 434

49. Haywood, K. L., Brett, J., Tutton, E., \& Staniszewska, S. (2017). Patient-reported outcome measures in older people with hip fracture: A systematic review of quality and acceptability. Quality of Life Research, 26(4), 799-812. https://doi.org/10.1007/ s11136-016-1424-1

50. Michalowsky, B., Xie, F., Kohlmann, T., Gräske, J., Wübbeler, M., Thyrian, J. R., \& Hoffmann, W. (2020). Acceptability and validity of the EQ-5D in patients living with Dementia. Value in Health, 23(6), 760-767. https://doi.org/10.1016/j.jval.2020.01.022

51. Menn, P., Weber, N., \& Holle, R. (2010). Health-related quality of life in patients with severe COPD hospitalized for exacerbationscomparing EQ-5D, SF-12 and SGRQ. Health and Quality of Life Outcomes, 8(1), 39. https://doi.org/10.1186/1477-7525-8-39

52. Kaambwa, B., Bryan, S., \& Billingham, L. (2012). Do the methods used to analyse missing data really matter? An examination of data from an observational study of Intermediate Care patients. BMC Research Notes, 5(1), 330. https://doi.org/10.1186/ 1756-0500-5-330

53. Joundi, R. A., Rebchuk, A. D., Field, T. S., Smith, E. E., Goyal, M., Demchuk, A. M., Dowlatshahi, D., Poppe, A. Y., Williams, D. J., Mandzia, J. L., Buck, B. H., Jadhav, A. P., Pikula, A., Menon, B. K., \& Hill, M. D. (2021). Health-related quality of life among patients with acute ischemic stroke and large vessel occlusion in the ESCAPE trial. Stroke, 52(5), 1636-1642. https://doi.org/10. 1161/STROKEAHA. 120.033872

54. Davis, J. C., Liu-Ambrose, T., Richardson, C. G., \& Bryan, S. (2013). A comparison of the ICECAP-O with EQ-5D in a falls prevention clinical setting: Are they complements or substitutes? Quality of Life Research, 22(5), 969-977. https://doi.org/10.1007/ s11136-012-0225-4

55. van Laar, C., Kievit, P. C., \& Noyez, L. (2015). Surgical aortic valve replacement in patients older than 75 years: Is there really a quality of life benefit? Netherlands Heart Journal, 23(3), 174179. https://doi.org/10.1007/s12471-015-0660-2

56. Feng, Y., Parkin, D., \& Devlin, N. J. (2014). Assessing the performance of the EQ-VAS in the NHS PROMs programme. Quality of Life Research, 23(3), 977-989. https://doi.org/10.1007/ s11136-013-0537-z

57. Lapin, B. R. (2020). Considerations for reporting and reviewing studies including health-related quality of life. Chest, 158(1S), S49-S56. https://doi.org/10.1016/j.chest.2020.03.007

58. Little, R. J., \& Rubin, D. B. (2002). Statistical analysis with missing data (Wiley series in probability and statistics). Wiley.

59. Coens, C., Pe, M., Dueck, A. C., Sloan, J., Basch, E., Calvert, M., Campbell, A., Cleeland, C., Cocks, K., Collette, L., \& Devlin, N. (2020). International standards for the analysis of quality-of-life 
and patient-reported outcome endpoints in cancer randomised controlled trials: recommendations of the SISAQOL Consortium. The Lancet Oncology, 21(2), e83-e96. https://doi.org/10.1016/ S1470-2045(19)30790-9

60. Madley-Dowd, P., Hughes, R., Tilling, K., \& Heron, J. (2019). The proportion of missing data should not be used to guide decisions on multiple imputation. Journal of Clinical Epidemiology, 110, 63-73. https://doi.org/10.1016/j.jclinepi.2019.02.016

61. Hardy, S. E., Allore, H., \& Studenski, S. A. (2009). Missing data: A special challenge in aging research. Journal of the American Geriatrics Society, 57(4), 722-729. https://doi.org/10.1111/j. 1532-5415.2008.02168.x

62. Pérez-Ros, P., \& Martínez-Arnau, F. M. (2020). EQ-5D-3L for assessing quality of life in older nursing home residents with cognitive impairment. Life, 10(7), 100. https://doi.org/10.3390/life1 0070100

63. Pérez-Ros, P., Vila-Candel, R., Martin-Utrilla, S., \& MartínezArnau, F. M. (2020). Health-related quality of life in communitydwelling older people with cognitive impairment: EQ-5D-3L measurement properties. Journal of Alzheimer's disease, 77(4), 1523-1532. https://doi.org/10.3233/JAD-200806

64. Davis, J. C., Best, J. R., Dian, L., Khan, K. M., Hsu, C. L., Chan, W., Winnie Cheung, W., \& Liu-Ambrose, T. (2017). Are the EQ5D-3L and the ICECAP-O responsive among older adults with impaired mobility? Evidence from the Vancouver falls prevention cohort study. Quality of Life Research, 26(3), 737-747. https://doi. org/10.1007/s11136-016-1487-z

65. de Leeuw, E. D. (2001). Reducing missing data in surveys: an overview of methods. Quality and Quantity, 35(2), 147-160. https://doi.org/10.1023/A:1010395805406

66. OECD. (2013). OECD guidelines on measuring subjective wellbeing. OECD.
67. Bhadhuri, A., Kind, P., Salari, P., Jungo, K. T., Boland, B., Byrne, S., Hossmann, S., Dalleur, O., Knol, W., Moutzouri, E., \& O'Mahony, D. (2020). Measurement properties of EQ-5D-3L and EQ-5D-5L in recording self-reported health status in older patients with substantial multimorbidity and polypharmacy. Health and Quality of Life Outcomes, 18(1), 317. https://doi.org/ 10.1186/s12955-020-01564-0

68. Devlin, N., Brazier, J., Pickard, A. S., \& Stolk, E. (2018). 3L, 5L, What the L? A NICE conundrum. PharmacoEconomics, 36(6), 637-640. https://doi.org/10.1007/s40273-018-0622-9

69. Rolstad, S., Adler, J., \& Rydén, A. (2011). Response burden and questionnaire length: Is shorter better? A review and meta-analysis. Value in Health, 14(8), 1101-1108. https://doi.org/10.1016/j. jval.2011.06.003

70. Kunz, S. (2010). Psychometric properties of the EQ-5D in a study of people with mild to moderate dementia. Quality of Life Research, 19(3), 425-434. https://doi.org/10.1007/ s11136-010-9600-1

71. Tidermark, J., Zethraeus, N., Svensson, O., Törnkvist, H., \& Ponzer, S. (2002). Femoral neck fractures in the elderly: Functional outcome and quality of life according to EuroQol. Quality of Life Research, 11(5), 473-481. https://doi.org/10.1023/a:10156 32114068

Publisher's Note Springer Nature remains neutral with regard to jurisdictional claims in published maps and institutional affiliations. 